
\title{
28 Research Square \\ miR-206 regulates a metabolic switch in nasopharyngeal carcinoma by suppressing HK2 expression
}

Chunying Luo

Guangxi medical university

Min Liu

Shanghai 6th Peoples Hospital Affiliated to Shanghai Jiaotong University School of Medicine Jianwei Zhang

Shanghai 6th Peoples Hospital Affiliated to Shanghai Jiaotong University School of Medicine

\section{Guoqiang Su}

Guangxi medical University

Zhonghua Wei ( $\nabla$ weizhonghua365@163.com )

Shanghai 6th Peoples Hospital Affiliated to Shanghai Jiaotong University School of Medicine https://orcid.org/0000-0003-0011-2347

\section{Research article}

Keywords: miR-206, Glycolysis, HK2, Nasopharyngeal Carcinoma

Posted Date: July 22nd, 2020

DOl: https://doi.org/10.21203/rs.3.rs-43672/v1

License: (c) (i) This work is licensed under a Creative Commons Attribution 4.0 International License. Read Full License 


\section{Abstract}

Background: Many studies have shown that microRNAs play key functions in nasopharyngeal carcinoma proliferation, invasion and metastasis. However, whether the dysregulated level of miRNAs contributes to the metabolic shift in nasopharyngeal carcinoma is not completely understood.

Objectives: This study was conducted to explore the expression and function of miR-206 in nasopharyngeal carcinoma.

Methods: miR-206 expression level was examined by real-time PCR. miR-206 inhibitor, mimics, and scrambled control were transiently transfected into nasopharyngeal carcinoma cells and their effects on colony formation, glucose uptake, and lactate secretion were observed in vitro. Moreover, the relationship between the levels of miR-206 and HK2 was examined by luciferase reporter and assay western blot.

Results: In our study, we reported downregulation of miR-206 expression leads to metabolic change in nasopharyngeal carcinoma cells. miR-206 controls this function by enhancing HK2 expression. The enhancement of aerobic metabolism activity induced by miR-206 leads to the rapid proliferation of nasopharyngeal carcinoma cells.

Conclusions: Our data demonstrated that miR-206 was involved in the regulation of Warburg effect in nasopharyngeal carcinoma by suppressing HK2 expression.

\section{Introduction}

A major source of cellular energy and new cell mass is glucose [1]. Glucose uptake in cancer tissues is obviously different from that in normal tissues. Normal tissues rely mainly on mitochondrial oxidative phosphorylation to produce ATP for cellular events, which is a more efficient energy produce event than glycolysis. However, cancer tissues rely primarily on aerobic glycolysis, through a phenomenon known as the "Warburg effect" [2]. Aerobic glycolysis confers cancer cells a selective advantage that results in their rapid growth. Increasing scientific evidence that shift of metabolism in cancer tissues is a potential therapeutic target [3].

MicroRNA (miRNAs) are a small group of endogenous non-coding RNAs, which are involved in regulating gene expression level through a sequence-specific manner [4]. Mature microRNAs negatively control their downstream target genes at post-transcriptional level by imperfect complementary binding to the $3^{\prime}$ untranslated region (UTR), leading to either translational repression or mRNA degradation [5]. MicroRNAs are known to be key regulators of target gene expression in diverse biological events, such as cell growth, apoptosis and metabolism.

MicroRNAs are known to be key regulators of cellular physiological processes, such as cell growth, cell metabolism, apoptosis [6, 7]. A large number of miRNAs, when aberrantly expressed, play important roles in cancer progression. Recently, many microRNAs have been reported as key regulators of aerobic 
metabolism. For example, miR-181a regulates a metabolic switch by regulating the PTEN/AKT pathway in colon cancer cells [8], whereas miR-424 is involved in metabolic reprogramming by suppressing the expression level of IDH3a in cancer-associated fibroblasts [9]. microRNA-33a and microRNA-33b play key functions in fatty acid catabolism and cholesterol homeostasis [10]. microRNA-495 regulates metabolic change by targeting Glut1 in glioma cells [11]. Knocking down of microRNA-182 is reported to enhance glucose consumption and glycolysis [12]. miR-206 is a class of "myomiRs" which is involved in heart disease, skeletal muscle development, Alzheimer's disease, and chronic obstructive pulmonary disease. However, recently the role of miR-206 in tumorigenesis received scrutiny. Previous study showed that miR206 inhibited tumor cell growth, invasion and cell apoptosis. However, whether miR-206 is involved in cancer cell metabolism remains unclear.

In our study, we demonstrated that miR-206 expression level was decreased in nasopharyngeal carcinoma cells. The reduction of miR-206 promotes glycolysis as measured by enhancing in glucose consumption rate and lactate secretion.

\section{Materials And Methods}

\section{Cell culture, reagents and clinical samples}

NP69, C666-1, HNE-1, CNE-1, and 293T cells were maintained in Dulbecco's modified Eagle medium with $10 \%$ fetal bovine serum (FBS, Invitrogen, California) and 100 units/ml penicillin/streptomycin. Primary antibodies against actin and HK2 were purchased from Santa Cruz Biotechnology. A total of five nasopharyngeal carcinoma and paired normal adjacent tissues at least $5 \mathrm{~cm}$ away from the tumor sites were acquired from each patient by biopsy prior to any treatment. All human patient samples were collected with informed consents from the patients, and the experiments were approved by the ethics committee of Shanghai Jiaotong University.

\section{Transfections}

microRNA-206 inhibitor(5'-ccacaugcuucuuuauauuccaua-3'), microRNA-206 mimics(5'uggaauguaaggaaguguguaa-3'), and scrambled siRNAs( $5^{\prime}$-ugggcguauagacguguuacac- $\left.3^{\prime}\right)$ were ordered from GenePharma (Shanghai, China). For the transient transfection, the cells were plated at $70 \%$ confluency and transfected with the indicated RNA molecules at a final concentration of $40 \mathrm{nM}$ with the PEl reagent.

\section{Quantitative real-time PCR}

Total RNA was extracted from cells with TRIzol reagent as described in the manufacturer's instructions. Reverse transcription of microRNA and mRNA was performed with the TaqMan qPCR miRNA assay kit. RT-qPCR detection of miR-206 was done with SYBR Green qPCR Supermix. U6 was used as an internal loading control.

\section{Western blot analysis}


The Cells were lysed by RIPA buffer. Total protein $(30 \mathrm{~g})$ was resolved using $8 \%$ SDS-PAGE and then transferred into nitrocellulose (NC) membrane. After blocking with $5 \%$ non-fat dried milk for $1 \mathrm{~h}$, the NC membrane was incubated with the primary antibody at $4^{\circ} \mathrm{C}$ overnight. After rinsing three times for $10 \mathrm{~min}$ each, the membrane was incubated with the secondary antibody for $1 \mathrm{~h}$ at room temperature. After washing with TBST three times, the signals were detected with the enhanced chemiluminescence (ECL) kit.

\section{Dual-luciferase reporter assay}

Dual-luciferase reporter assay was used to confirm whether miR-206 downregulates Glut1 via binding to the 3' UTR region. HEK293T cells were seeded in a 48-well plate at 70-80\% confluency. After cotransfection with either $20 \mathrm{nM}$ of miR-206 mimics or scrambled controls and $40 \mathrm{ng}$ of pGL-Glut1-3'UTRwt or pGL-Glut1-3'UTR-mut, cells were harvested to measure the luciferase activity with Dual Luciferase Assay Kit (Promega, Madison, WI). Experiments of luciferase activity assay were performed independently in triplicates.

\section{Cell proliferation assay}

Cells were plated at a density of 5000 cells/well in 96-well plates and transfected with the indicated RNA molecules. After culturing in DMEM without serum for $6 \mathrm{~h}$, the cells were allowed to recover in the culture medium for $48 \mathrm{~h}$. After incubation for $48 \mathrm{~h}$, the absorbance at $450 \mathrm{~nm}$ was measured with the Cell Counting kit-8 assay kit (CCK8) (DOJINDO. Tabaru, Japan). The cell survival rate was calculated using the equation:

survival rate $(\%)=\left(A_{\text {sample }}-A_{\text {blank }}\right) /\left(A_{\text {control }}-A_{\text {blank }}\right) \times 100$.

\section{Measurement of glucose uptake and extracellular lactate secretion}

The miR-206 inhibitor or mimics were transfected into nasopharyngeal carcinoma cells in serum-free DMEM. After incubation for $48 \mathrm{~h}$, the medium was collected. Lactate production was determined using the lactate assay kit (Sigma-Aldrich). Glucose uptake rate was determined using a colorimetric assay kit (Glucose Uptake Colorimetric Assay Kit, BioVision).

\section{Statistical analysis}

Statistical analyses were carried out with GraphPad Prism software. Data are presented as the mean \pm SD. Results were considered to be statistically significant if $p<0.05$.

\section{Results}

\section{miR-206 is downregulated in nasopharyngeal carcinoma}


To examine the expression level of miR-206 in nasopharyngeal carcinoma, we analyzed the expression profile of microRNAs in nasopharyngeal carcinoma from the previously published dataset (Gene expression omnibus accession GSE43039) and found that miR-206 was downregulated in nasopharyngeal carcinoma (Fig 1. A, B). To analyze the microRNA expression levels in human nasopharyngeal carcinoma, we performed qRT-PCR to measure the miR-206 level in five matched clinical sample pairs. The data indicated that miR-206 expression was markedly reduced in human nasopharyngeal carcinoma tissues compared to the adjacent normal tissues (Fig 1. C). In addition, we examined the expression level of miR-206 in human nasopharyngeal carcinoma cell lines. As expected, miR-206 expression level was significantly reduced in nasopharyngeal carcinoma cell lines C666-1, HNE1, and CNE-1 compared with the normal osteoblastic cell line, NP69 (Fig 1. D).

\section{miR-206 inhibits proliferation of nasopharyngeal carcinoma cells}

To explore the biology of miR-206 in the nasopharyngeal carcinoma cells, C666-1 cells were transfected with miR-206 inhibitor, miR-206 mimics, or scrambled siRNA at a final concentration of $40 \mathrm{nM}$. The qRTPCR assay showed that miR-206 expression was significantly enhanced in the mimic-transfected cells and decreased in the inhibitor-transfected cells (Fig 2. A). The CCK8 assay showed that the cell proliferation rate was increased in miR-206 knockdown cells compared with the scrambled group (Fig 2. B). As expected, overexpression of miR-206 reduced cell growth (Fig 2. B). In addition, the colony formation assay suggested that loss-of-function of miR-206 increased colony formation in C666-1 cells. In contrast, gain-of function of miR-206 reduced colony formation in C666-1 cells (Fig 2. C, D). To further confirm the cell proliferation data and to examine the role of miR-206 in the regulation the cell cycle, we performed flow cytometry after transfecting cells with miR-206 mimics. Overexpression of the miR-206 led to significant reduction in the number of cells in S-phase (Fig 2. E). Taken together, these results indicate that miR-206 functions as a tumor suppressor in C666-1 cells.

\section{miR-206 induces a metabolic switch in C666-1 and HNE-1 cells}

To explore the role of miR-206 in the metabolism of nasopharyngeal carcinoma cells, we tested the effect of miR-206 on glucose uptake rate and lactate secretion. The data showed that ectopic expression of miR-206 in C666-1 and HNE-1 cells transfected with miR-206 mimics reduced lactate secretion and glucose uptake rate (Fig 3. A, B). In contrast, knockdown of miR-206 in C666-1 and HNE-1 cells with miR206 inhibitors increased the lactate secretion and glucose uptake rate (Fig 3. A, B). Glut1, PFKL, and PKM2 are key glycolysis genes. Western blot analysis showed that knockdown of miR-206 increased the expression of Glut1, PFKL, and PKM2 (Fig 3. C).. To further explore whether the metabolic switch induced by miR-206 knockdown was required for the tumor cell growth, we treated miR-206-downregulated C666-1 cells with 2-deoxyglucose (2-DG), which inhibits glycolysis by blocking hexokinase activity. We used CCK8 assay to examine the cell proliferation rates. The results showed that inhibition of glycolysis by 2DG was sufficient to reduce cell growth (Fig 3. D). In conclusion, these data showed that increased glycolysis induced by miR-206 is required for the increased tumor cell growth.

\section{miR-206 downregulates HK2 expression by binding its 3'UTR}


To further elucidate the molecular mechanism by which miR-206 increases glycolysis, we used miRanda to predict the downstream target genes of miR-206 based on the $3^{\prime}$ UTR. We found that the $3^{\prime}$ UTR region of Hexokinase 2 (HK2) was a predicted target of miR-206 (Fig 4. A). HK2 plays an essential role in cancer growth, metastasis and survival. To validate the regulation of HK2 expression by miR-206, we performed western blot and the results showed that overexpression of miR-206 results in downregulation of HK2 expression (Fig 4. B). Conversely, transfection with miR-206 inhibitors resulted in an elevation of endogenous HK2 expression (Fig 4. C). Next, to examine whether miR-206 directly binds the 3'UTR region of HK2, we carried out dual luciferase reporter assay. The data demonstrated that miR-206 suppressed luciferase activity in the wild-type seed region group. However, the suppression of the luciferase activity was rescued in the mutant group (Fig 4. D). In conclusion, the data indicated that miR-206 downregulates HK2 expression through direct binding its 3'UTR.

\section{Discussion}

MicroRNAs have emerged as key regulators of different biological events, including cell proliferation, invasion, differentiation, apoptosis and cell metabolism. Therefore, a potential therapeutic strategy would be to target onco-miRNAs or restore the expression of tumor suppressor miRNAs. Recently, several miRNAs, such as miR-let-7a, miR148b, miR-424, and miR-181a were identified as regulators of glycolysis $[8,9,13,14]$. Previous reports showed that dysregulation of miR-206 was associated with different cancers. For example, miR-206 expression is lower in hepatocellular carcinoma. miR-206 was shown to suppress cell proliferation and promote apoptosis. Lower expression of miR-206 is associated with poor clinical outcome in patients with malignant astrocytomas. Downregulation of miR-206 is a potent prognostic marker for patients with gastric cancer. These results showed that miR-206 can inhibit cell proliferation by targeting VEGFA [15], inhibit cell migration via targeting PFKFB3 [16], reverse cisplatin resistance and EMT by targeting MET [17], and induce cancer cell cycle arrest at G1/G0 phase by targeting FMN2 [18]. However, the expression profile of miR-206 and its biological role in nasopharyngeal carcinoma remains unknown.

In this study, we reported that miR-206 expression was downregulated in nasopharyngeal carcinoma compared to adjacent normal tissues. This is consistent with the findings of a previous report which showed that miR-206 was downregulated in radioresistant nasopharyngeal carcinoma cells, and thus, suppressed the IGF1 and PI3K/AKT pathways. Previous studies have showed that miR-206 acts as a tumor suppressor to repress cell growth in clear-cell renal cell carcinoma, cervical cancer and hepatocellular carcinoma [19-21]. Consistent with these findings, our CCK8 assay data indicated that miR-206 decreased cell growth rate. The molecular mechanism underlying enhanced glycolysis in cancer cells is complicated and not yet clearly understood. Bioinformatic analysis was performed to predict the targets of miR-206. HK2 was selected as a potential downstream target of miR-206 with possible involvement in the function of miR-206. HK2 is highly expressed in many cancers and is required for tumor glycolysis and initiation. We reported that miR-206 plays a key role in glycolysis of nasopharyngeal carcinoma by directly targeting HK2 expression. Gain of miR-206 function decreases lactate secretion and glucose uptake rate, while loss of miR-206 function increases glucose uptake rate and lactate 
secretion. The inhibition of glycolysis can transform tumor cells into forms that are sensitive to chemotherapy or immunotherapy. Thus, novel diagnostic methods and new drugs can be developed by understanding the features of glycolysis. The enhanced glycolysis induced by downregulation of miR-206 can be targeted for treatment of nasopharyngeal carcinoma.

In conclusion, our data show that miR-206 was markedly downregulated in nasopharyngeal carcinoma, thus leading to enhanced glycolysis in cancer cells. This altered glycolysis is required for rapid cancer cell growth. These data identify miR-206 as a potential therapeutic target for treatment of nasopharyngeal carcinoma.

\section{Declarations}

\section{Ethics approval and consent to participate}

Not applicable.

\section{Consent for publication}

Not applicable.

\section{Competing interests}

The authors declare that they have no competing interests.

\section{Availability of data and materials}

The data that support the findings of this study are available from the corresponding author upon reasonable request.

\section{Authors' contributions}

C.L and M.L designed and performed most of the experiment; J.L analysed the data; G.S and Z.W designed some of the experiment and wrote the manuscript. All authors read this manuscript.

\section{Acknowledgements}

Not applicable.

\section{Abbreviations}

$\mathrm{PCR}=$ Polymerase chain reaction; HK2= Hexokinase $2 ; \mathrm{ATP}=$ Adenosine triphosphate; $\mathrm{UTR}=$ untranslated region; $A K T=$ Protein kinase $B ; P T E N=P h o s p h a t a s e$ and tensin homolog; IDH3a= Isocitrate Dehydrogenase Subunit Alpha; Glut1 = Glucose transporter 1; PFKL= 6-phosphofructokinase, liver type; PKM2= Pyruvate kinase muscle isozyme M2; VEGFA= vascular endothelial growth factor $A$ 


\section{References}

1. Cairns RA, Harris IS, Mak TW. Regulation of cancer cell metabolism. Nature reviews Cancer. 2011;11:85-95.

2. Hsu PP, Sabatini DM. Cancer cell metabolism: Warburg and beyond. Cell. 2008;134:703-7.

3. Kroemer G, Pouyssegur J. Tumor cell metabolism: cancer's Achilles' heel. Cancer cell. 2008;13:47282.

4. Treiber T, Treiber N, Meister G. Regulation of microRNA biogenesis and function. Thromb Haemost. 2012;107:605-10.

5. Hayes J, Peruzzi PP, Lawler S. MicroRNAs in cancer: biomarkers, functions and therapy. Trends in molecular medicine. 2014;20:460-9.

6. Wang Y, Lee CG. MicroRNA and cancer-focus on apoptosis. Journal of cellular molecular medicine. 2009;13:12-23.

7. Xu P, Li Y, Zhang H, Li M, Zhu H. MicroRNA-340 Mediates Metabolic Shift in Oral Squamous Cell Carcinoma by Targeting Glucose Transporter-1. J Oral Maxillofac Surg. 2016;74:844-50.

8. Wei Z, Cui L, Mei Z, Liu M, Zhang D. miR-181a mediates metabolic shift in colon cancer cells via the PTEN/AKT pathway. FEBS Lett. 2014;588:1773-9.

9. Zhang D, Wang Y, Shi Z, Liu J, Sun P, Hou X, Zhang J, Zhao S, Zhou BP, Mi J. Metabolic reprogramming of cancer-associated fibroblasts by IDH3alpha downregulation. Cell Rep. 2015;10:1335-48.

10. Davalos A, Goedeke L, Smibert P, Ramirez CM, Warrier NP, Andreo U, Cirera-Salinas D, Rayner K, Suresh U, Pastor-Pareja JC, Esplugues E, Fisher EA, Penalva LO, Moore KJ, Suarez Y, Lai EC, Fernandez-Hernando C. miR-33a/b contribute to the regulation of fatty acid metabolism and insulin signaling. Proceedings of the National Academy of Sciences of the United States of America. 2011;108:9232-7.

11. Nie S, Li K, Huang Y, Hu Q, Gao X, Jie S. miR-495 mediates metabolic shift in glioma cells via targeting Glut1. The Journal of craniofacial surgery. 2015;26:e155-8.

12. Hu JW, Sun P, Zhang DX, Xiong WJ, Mi J. Hexokinase 2 regulates G1/S checkpoint through CDK2 in cancer-associated fibroblasts. Cellular signalling. 2014;26:2210-6.

13. Ding X, Liu J, Liu T, Ma Z, Wen D, Zhu J. miR-148b inhibits glycolysis in gastric cancer through targeting SLC2A1. Cancer Med. 2017;6:1301-10.

14. Tang R, Yang C, Ma X, Wang Y, Luo D, Huang C, Xu Z, Liu P, Yang L. MiR-let-7a inhibits cell proliferation, migration, and invasion by down-regulating PKM2 in gastric cancer. Oncotarget. 2016;7:5972-84.

15. Cai Y, Li H, Zhang Y. Downregulation of microRNA-206 suppresses clear cell renal carcinoma proliferation and invasion by targeting vascular endothelial growth factor A. Oncol Rep. 2016;35:1778-86. 
16. Ge X, Lyu P, Cao Z, Li J, Guo G, Xia W, Gu Y. Overexpression of miR-206 suppresses glycolysis, proliferation and migration in breast cancer cells via PFKFB3 targeting. Biochem Biophys Res Commun. 2015;463:1115-21.

17. Chen QY, Jiao DM, Wang J, Hu H, Tang X, Chen J, Mou H, Lu W. miR-206 regulates cisplatin resistance and EMT in human lung adenocarcinoma cells partly by targeting MET. Oncotarget. 2016;7:24510-26.

18. Ren XL, He GY, Li XM, Men H, Yi LZ, Lu GF, Xin SN, Wu PX, Li YL, Liao WT, Ding YQ, Liang L. MicroRNA-206 functions as a tumor suppressor in colorectal cancer by targeting FMNL2. J Cancer Res Clin Oncol. 2016;142:581-92.

19. Wang Y, Tian Y. miR-206 Inhibits Cell Proliferation, Migration, and Invasion by Targeting BAG3 in Human Cervical Cancer. Oncol Res. 2018;26:923-31.

20. Heinemann FG, Tolkach Y, Deng M, Schmidt D, Perner S, Kristiansen G, Muller SC, Ellinger J. Serum miR-122-5p and miR-206 expression: non-invasive prognostic biomarkers for renal cell carcinoma. Clin Epigenetics. 2018;10:11.

21. Yunqiao L, Vanke H, Jun X, Tangmeng G. MicroRNA-206, down-regulated in hepatocellular carcinoma, suppresses cell proliferation and promotes apoptosis. Hepatogastroenterology. 2014;61:1302-7.

\section{Figures}


A

こ๐m

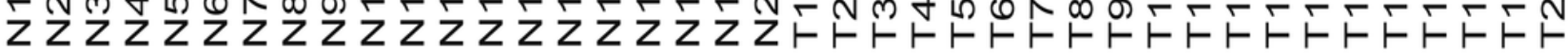

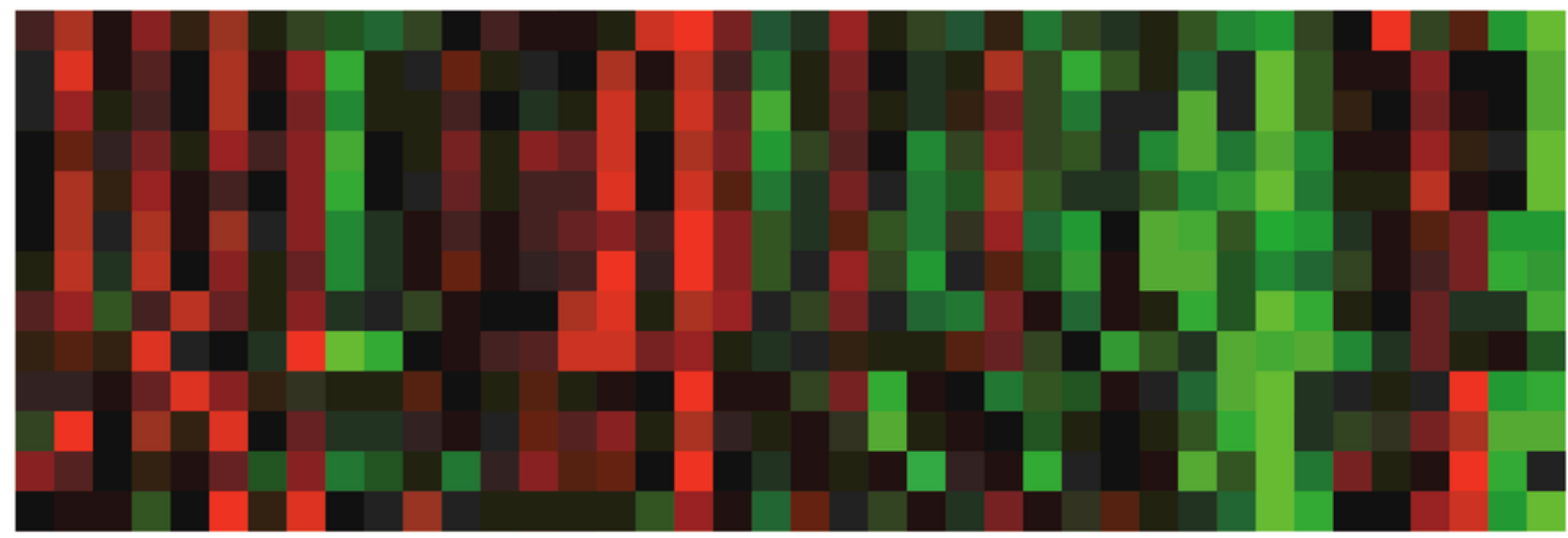

miR-302b

miR-10a

miR-217

miR-10b

miR-432

miR-31

miR-448

miR-206

miR-197

miR-146a

miR-452

miR-196b

miR-565
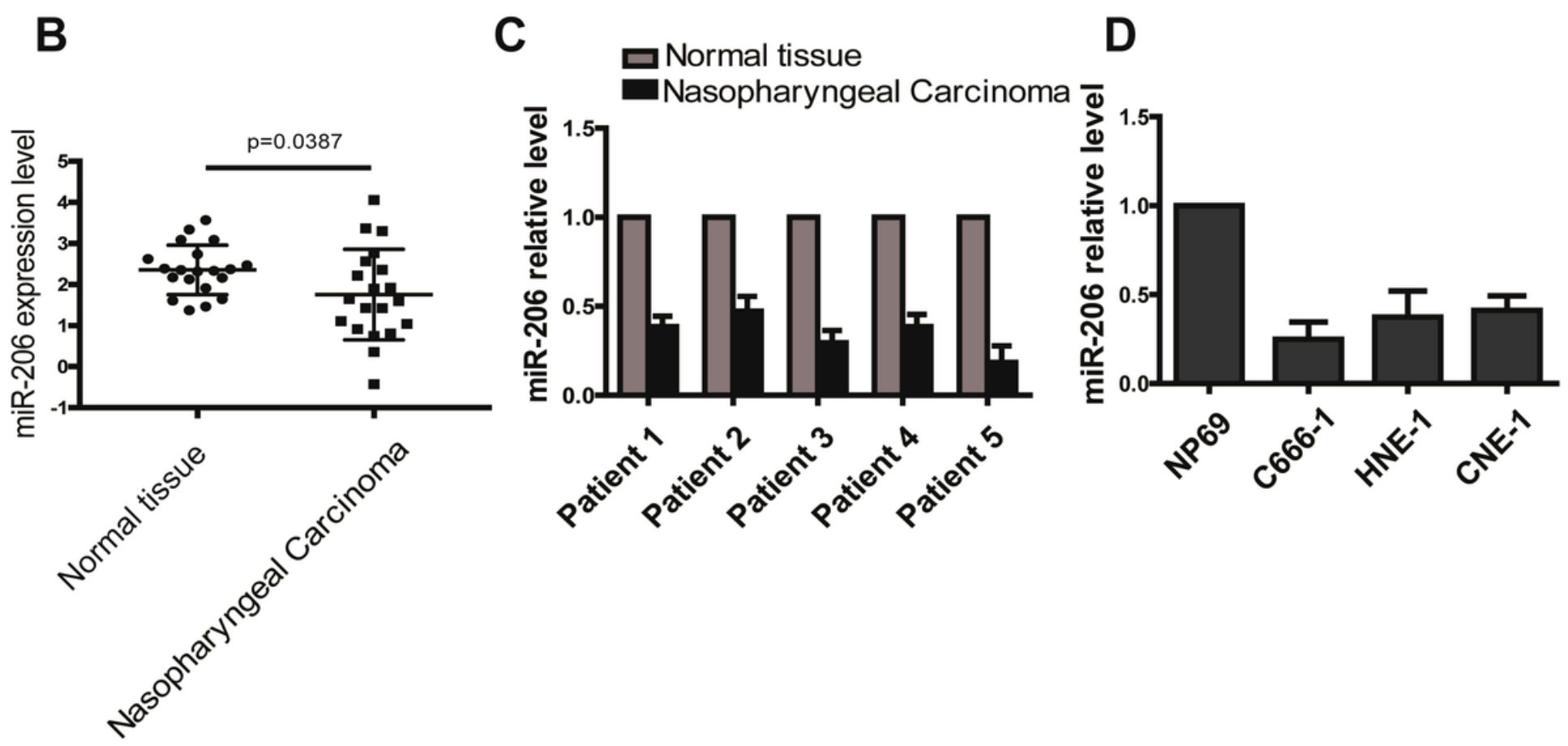

Figure 1

miR-206 is downregulated in nasopharyngeal carcinoma. (A) Heatmap showing the expression of selected microRNAs in nasopharyngeal carcinoma compared with normal tissues. (B) The microRNA expression assay reveals that miR-206 was significantly downregulated in nasopharyngeal carcinoma compared to normal tissue. (C) Real-time PCR analysis shows that miR-206 was significantly downregulated in nasopharyngeal carcinoma compared with normal tissues. (D) Real-time PCR analysis shows that miR-206 expression was significantly downregulated in nasopharyngeal carcinoma cell lines compared to the normal cell line. 
A
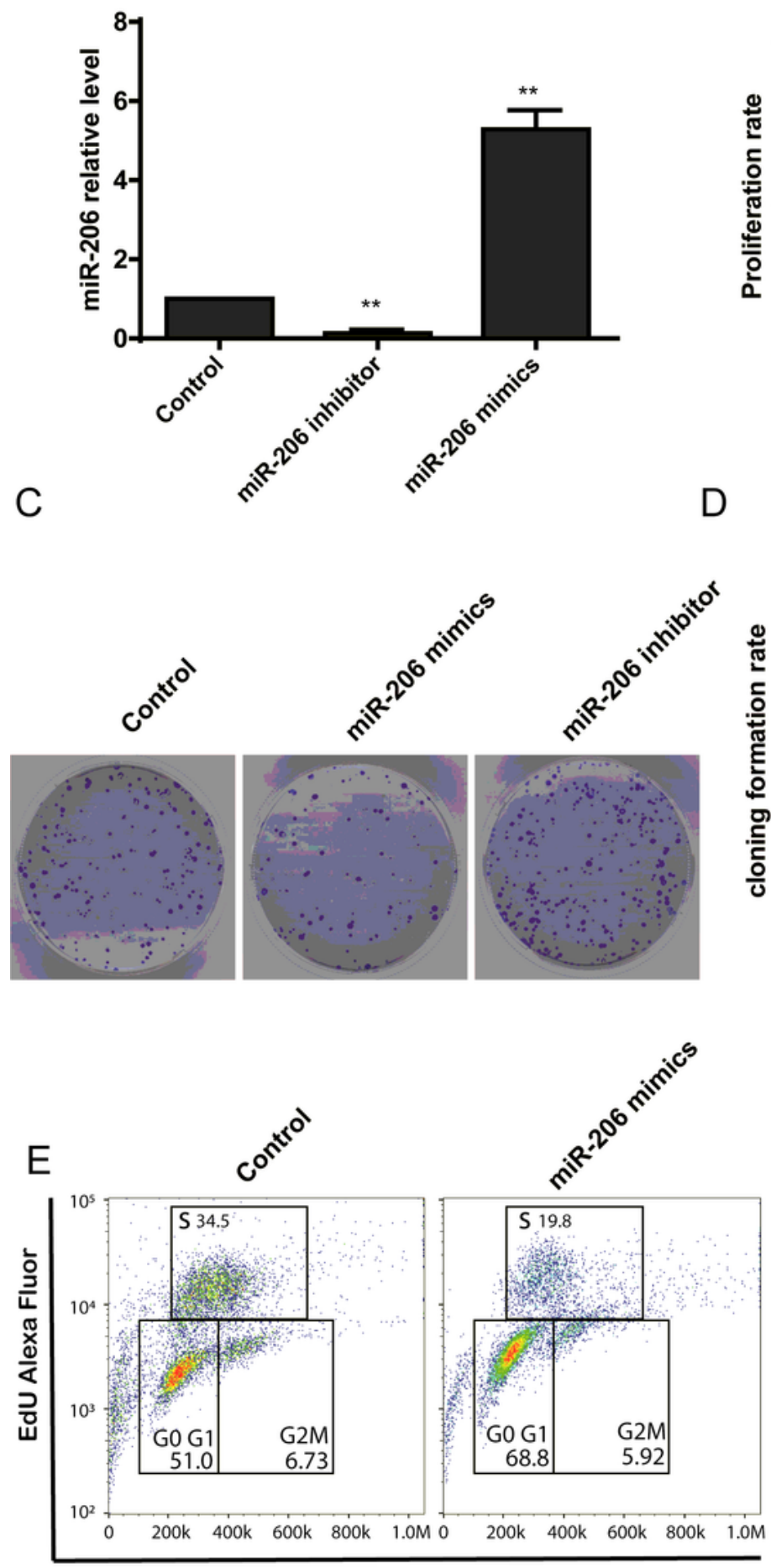

Fx Cycle Violet
B

$$
\begin{aligned}
& \text { Control } \\
& \text { miR-206 mimics } \\
& \text { miR-206 inhibitor }
\end{aligned}
$$

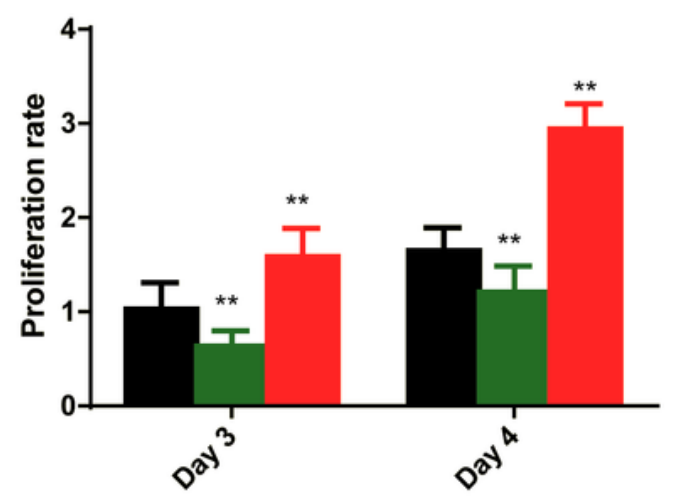

D
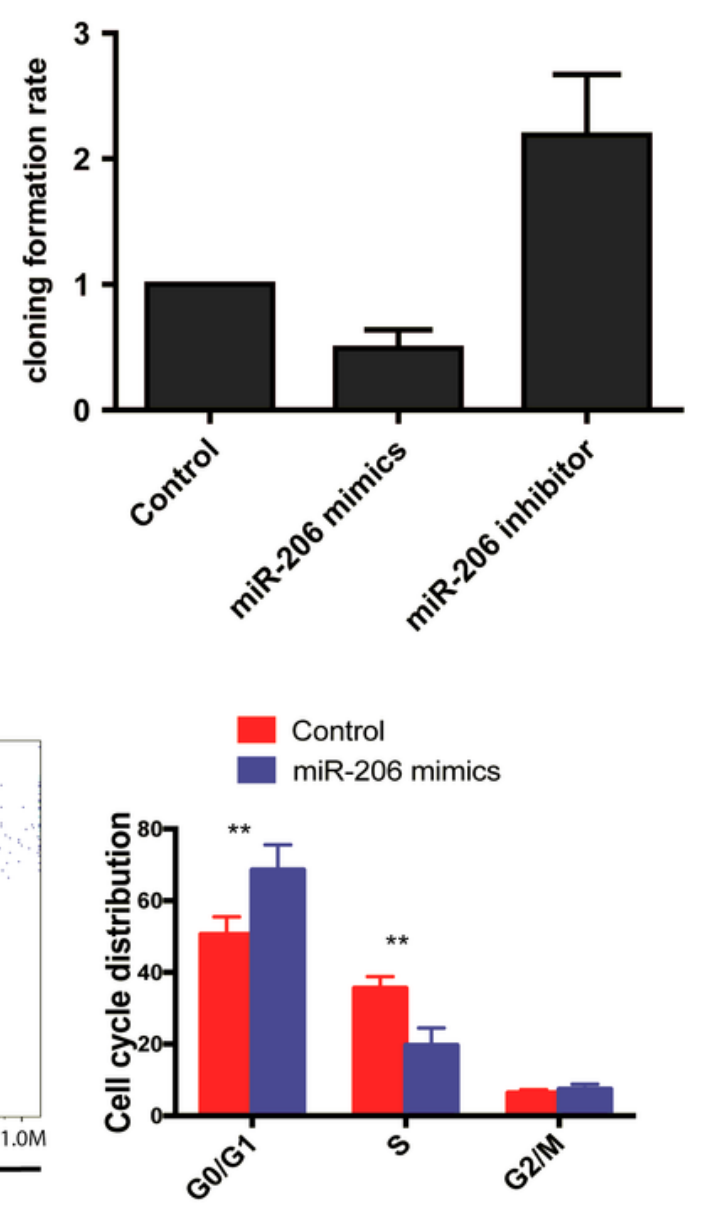

\section{Figure 2}

miR-206 inhibits the proliferation of nasopharyngeal carcinoma cells. (A) Real-time PCR analysis showing miR-206 levels in nasopharyngeal carcinoma cells transfected with the miR-206 mimics or the miR-206 inhibitor. (B) The CCK8 assay results show that the growth rate of miR-206-overexpressing nasopharyngeal carcinoma cells was reduced compared to control cells. Conversely, knockdown of miR206 in nasopharyngeal carcinoma cells enhanced cell growth. (C) and (D) The colony formation assay 
shows that knockdown of miR-206 enhanced colony formation in nasopharyngeal carcinoma cells, while overexpression of miR-206 decreased colony formation in nasopharyngeal carcinoma cells. (E) Cells were transfected with scramble or miR-206 mimics and cell cycle was analyzed by flow cytometry.

A

$\square$ Control

miR-206 inhibitor

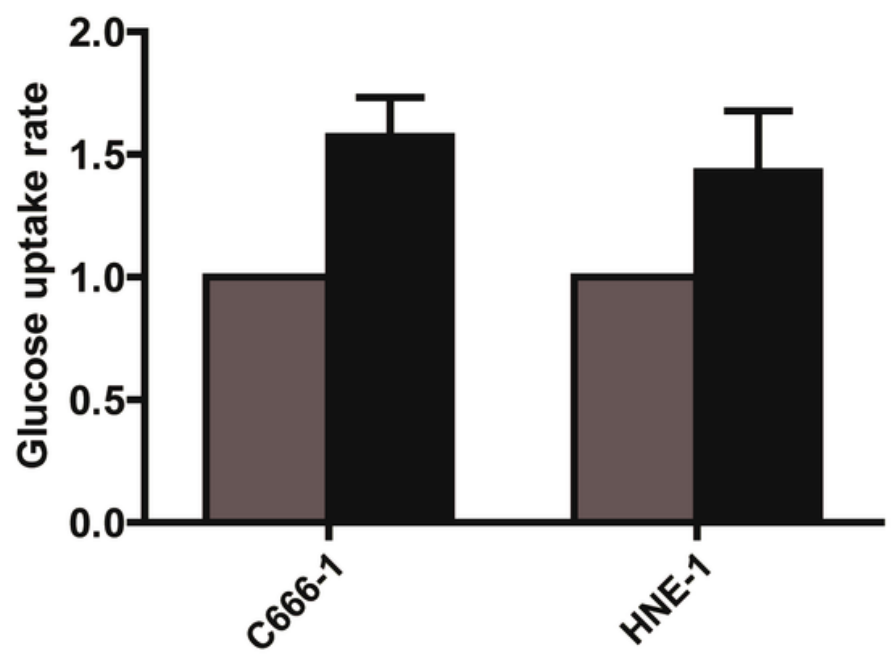

C

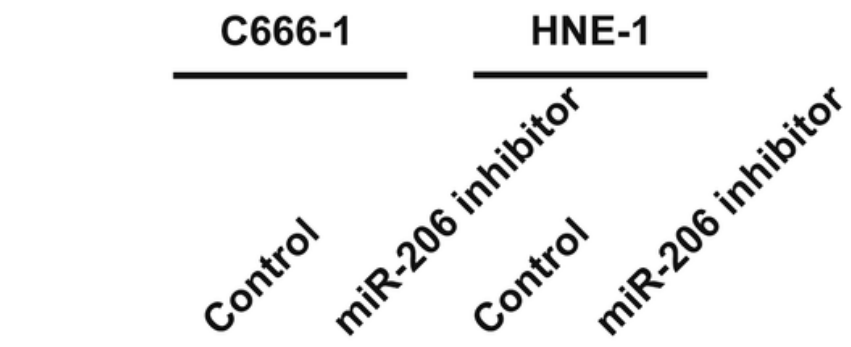

Glut1

PKM2

PFKL

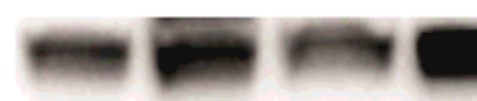

Actin
B
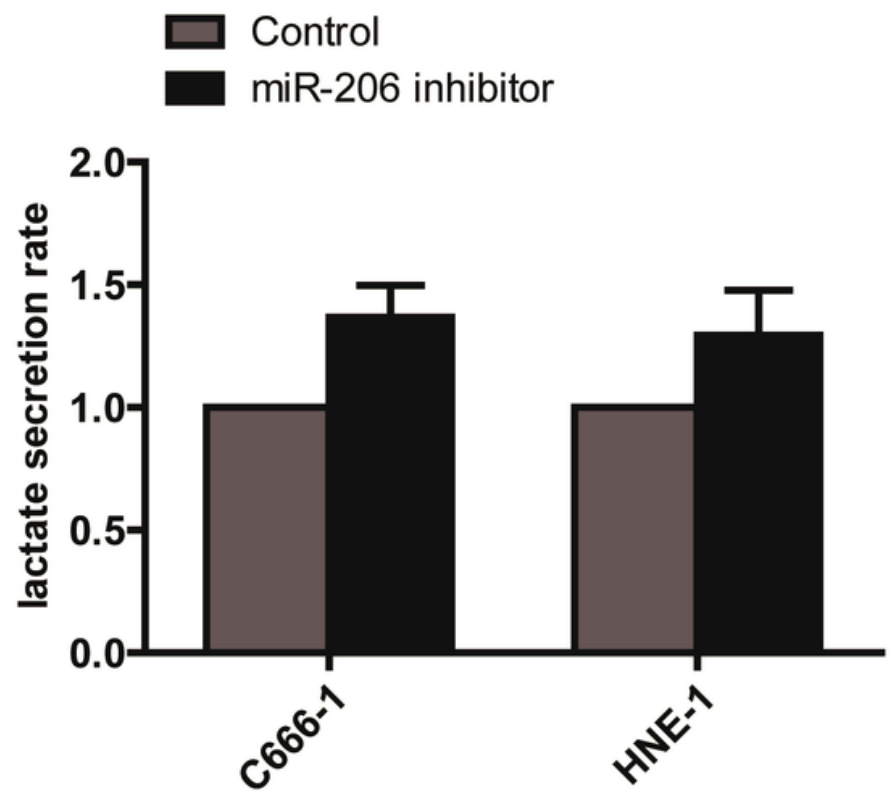

D 
expression of Glut1, PKM2, and PFKL. (D) The CCK8 assay shows that inhibition of glycolysis with 2-DG suppressed the enhanced proliferation induced by knockdown of miR-206.

A

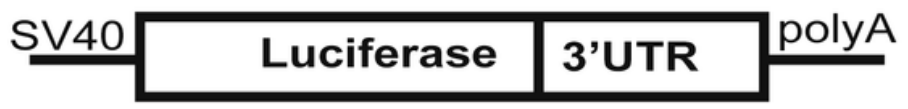

HK2-WT 5'-UCACAUGCAUCAUAACCAUUCCC-3' miR-206 3'-ggUGUGUGAAGGAAU-GUÁÁGG-5' HK2-mut 5'-ucACAUGCAUCAUAACCUUACGc-3'

C

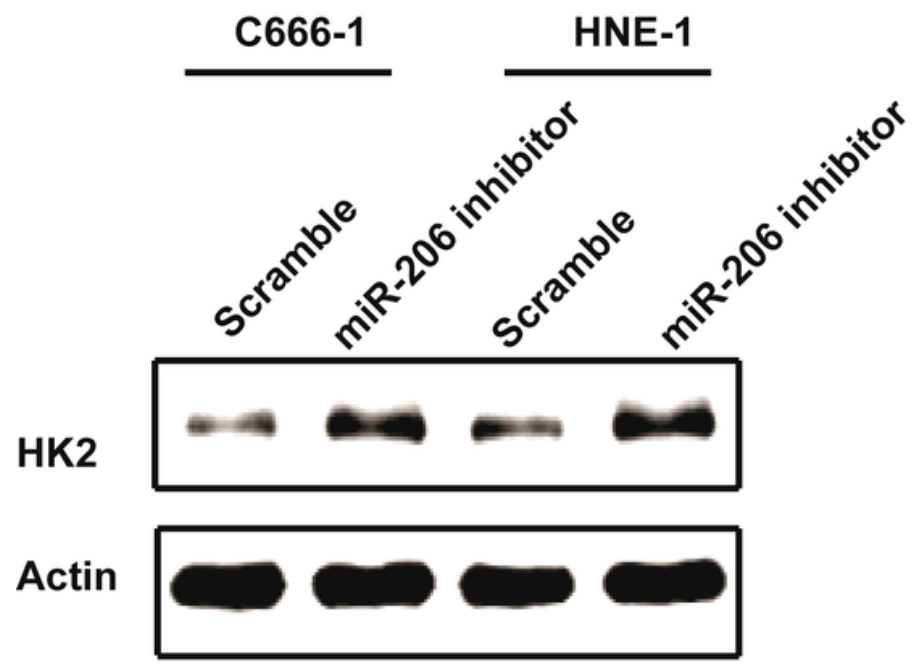

B

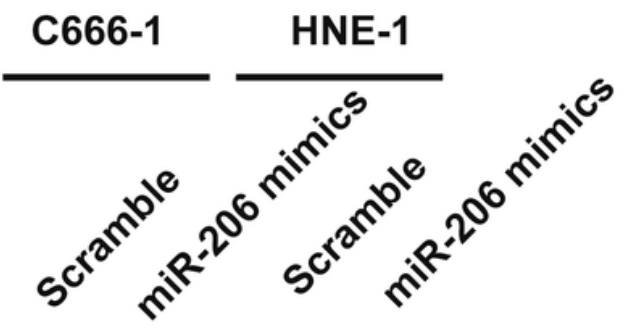

HK2

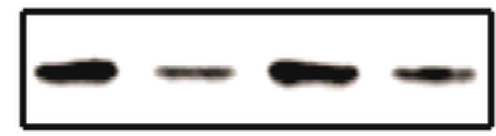

Actin

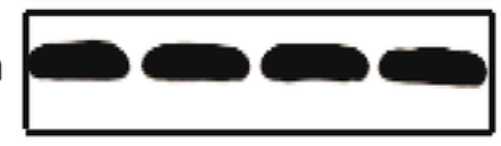

D

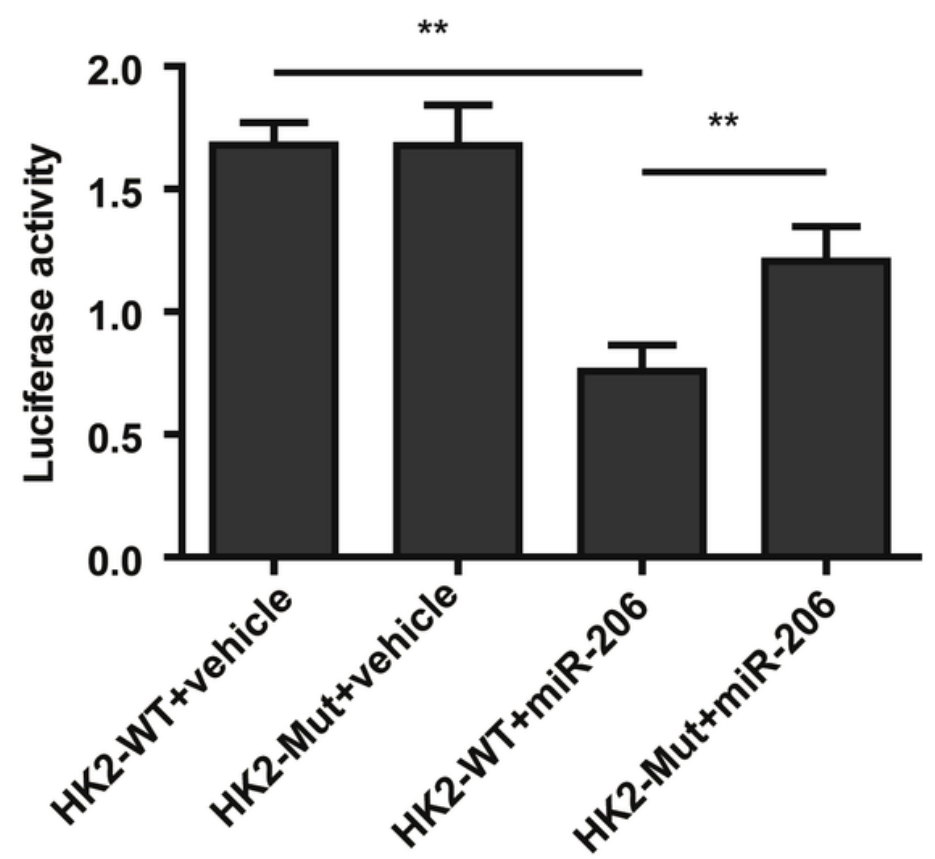

Figure 4

miR-206 suppresses expression of HK2. (A) Sequences of miR-206 and the potential binding sites at the 3 'UTR of HK2. (B) Western blot assay shows that overexpression of miR-206 suppressed the expression of HK2. (C) Western blot assay shows that knockdown of miR-206 increased the expression of HK2. (D) Dual-luciferase assay shows that miR-206 suppressed the luciferase activity of HK2-3'UTR wild-type group but not that of the HK2-3'UTR mutant group. 